\title{
Canadian Association of Neuropathologists
}

\author{
Abstracts of papers and cases presented at the \\ 37th Annual Meeting
}

\author{
October 22nd - 25th, 1997 \\ Ottawa, Ontario
}

The 37th annual meeting of the Canadian Association of Neuropathologists was held from October 22nd - 25th, at the Chateau Laurier Hotel in Ottawa, Ontario. Local arrangements were made by Dr Vital Montpetit.

The scientific session consisted of 19 platform presentations and 14 cases for diagnosis. The Royal College of Physicians and Surgeons of Canada speaker was Dr. Michael Noel Hart, MD, PhD, Professor of Pathology and Laboratory Medicine, University of Wisconsin Medical School. His talk was entitled "Immunology of the Blood-Brain Barrier". The Jerzy Olszewski lecturer was Dr. Antoine M. Hakim, Chairman, Division of Neurology, and Director, Neuroscience Research Institute, University of Ottawa/Ottawa General Hospital. His talk was entitled "Current Concepts and Future Prospects in Cerebral Ischemia and Stroke". 


\section{Abstracts of Papers Presented at the 37th Annual Meeting of the Canadian Association of Neuropathologists}

\section{PLATFORM PRESENTATIONS}

1.

\section{Blocking Mevalonate Synthesis is a Critical Step in the Mechanism of Lovastatin-Induced Medulloblastoma Apoptosis.}

W. WANG and R.J.B. MACAULAY (Department of Pathology, University of Saskatchewan, Saskatoon)

Surgery and radiotherapy for medulloblastomas is disabling, and only achieves about $50 \%$ five year survival. A potential new therapeutic strategy using lovastatin, a competitive inhibitor of 3hydroxy-3-methylglutaryl coenzyme A (HMG-CoA) reductase, has been studied in our laboratory. HMG-CoA reductase is a key ratelimiting enzyme in the mevalonate pathway, which normally leads to cholesterol biosynthesis and the production of non-steroidal mevalonate derivatives. Some of these products have been implicated in a number of growth-regulatory processes. Using medulloblastoma cell lines in vitro, we previously showed that blocking the mevalonate pathway with lovastatin first inhibits medulloblastoma proliferation, and then induces apoptosis in a dose and timedependent manner. Further characterization of the mechanism of lovastatin-induced apoptosis shows that: 1) mevalonate prevents lovastatin-induced apoptosis in lovastatin-pretreated cells; 2) this protection is time-dependent, since mevalonate fails to prevent lovastatin-induced apoptosis after longer lovastatin pretreatment; 3 ) mevalonate restores the lovastatin-arrested cell cycle, allowing $S$ phase entry; 4) the increase in HMG-CoA reductase mRNA levels induced by lovastatin is reversed by co-incubation with lovastatin and mevalonate. These results confirm that lovastatin inhibition of HMG-CoA reductase results in blockage of the mevalonate pathway, and demonstrate that blocking mevalonate production is a critical step in the mechanism of lovastatin-induced apoptosis.

2.

\section{Congenital CNS Tumors: Montreal Children's Hospital 1982-1997 Experience.}

K. MEAGHER-VILLEMURE, J.P. FARMER, J.L. MONTES, A.M. O'GORMAN (Montreal Children's Hospital and McGill University, Montreal)

Central nervous system tumors developing in utero or with presenting symptoms within the first 60 days after birth are extremely rare. We are describing the Montreal Children's Hospital experience of the past fifteen years. Twelve congenital tumors were studied. Ten babies presented with hydrocephalus, two cases had spinal cord involvement, one case had hemifacial spasm and two cases presented with other organ defects. Histologically, a variety of tumors were seen, benign or malignant. There were two choroid plexus papillomas of the lateral ventricles, one astrocytoma, one primitive neuroectodermal tumor, one ganglioglioma in the brainstem region, one medulloepithelioma and a metastasis from a
Wilm's tumor in the cerebellum, a rhabdoid tumor and an atypical teratoid rhabdoid tumor infiltrating the whole spinal cord, an embryonal tumor with neuroblastic and rhabdoid features in the cerebrum and one pinealoblastoma. The prognosis of these tumors is poor for undifferentiated and infiltrative types and relatively good for those well-differentiated and resectable. A review of the literature and pathogenesis of these tumors is discussed in regard to the genetic factors, familial occurrence, intrauterine infections and environmental factors.

\section{3.}

\section{Phenotypic Patterns of Synaptic Density in the Molecular Layer of the Dentate Gyrus in Adult Neurodegenerative Diseases and Normal Controls.}

Y. ROBITAILLE, H. DILHUYDY, J. GUEVARA, R. MENA, R. QUIRION and D. GAUVREAU (Institute of Geriatrics Research Centre and Department of Pathology, University of Montreal, Montreal)

The molecular layer of the dentate gyrus is a key synaptic relay station for perforant pathway inputs. The latter originate from the parasubiculum, which itself receives afferent fibres from many cortical areas and limbic structures. Using an anti-human synaptophysin Mab (EP-10), we compared patterns of dendritic synaptic innervation within basal, intermediate and apical layers of the molecular layer of the dentate gyrus. Brain tissues from Alzheimer's Disease (AD) $(n=17)$, non-AD demented $(n=9)$ and normal controls $(n=11)$ subjects were sampled in the fresh state according to a standardized protocol and were then fixed in paraformaldehyde $4 \%$ for at least $36 \mathrm{hrs}$. All sections were sampled from the posterior hippocampus adjacent to the lateral geniculate body. Synaptic densities (SD) were measured on immunoperoxidase-labelled sections within each specific dendritic layer using an image analyzer. In normal controls, SD were largely homogeneous. The highest levels were recorded in the basal and/or intermediate strata. In $\mathrm{AD}$, except for one case, the SD within intermediate and apical layers were significantly reduced. Finally, non$A D$ demented subjects showed patterns of $S D$ ranging from the normal to levels similar to those of AD brains. We conclude that $\mathrm{SD}$ changes measured by EP-10 immunocytochemistry in the molecular layer likely reflect heterogeneous patterns of perforant pathway deafferentation in demented subjects.

4.

Sudden Unexpected Death in an Adolescent With Periventricular and Subcortical Nodular Heterotopia (PNH and SNH).

A. HALIL, V.J.A. MONTPETIT, B. JOHNSTON and B. BECHARD (University of Ottawa and Office of the Chief Coroner, Ottawa)

Seizures are a well known cause of sudden unexpected death and are also common in patients with $\mathrm{PNH}$ and $\mathrm{SNH}$. We report 
a 16-year-old healthy female who complained only of mild asthma and whose only medication was the birth control pill. She was found dead in the morning, her body half on the bed and her head on the floor. Her mother carried her through an uncomplicated pregnancy, although she was induced prematurely due to an error in dates. Early developmental milestones were normal. She had some problems in school due to angry outbursts but, most recently, had started to improve scholastically. There is no family history of sudden unexplained death, seizure disorders or miscarriages. Autopsy findings included mild bilateral pulmonary edema, negative toxicology and bilateral PNH and bilateral calcified SNH. A review of the literature indicates that patients with both PNH and SNH are more likely to have seizures, have earlier onset seizures and have an increased prevalence of developmental delay when compared to patients with PNH alone. Both sporadic and genetic causes exist and pathogenic mechanisms for each will be reviewed. Given her autopsy findings and the natural course of her disease, a seizure appears to be the most likely cause of this patient's sudden death.

5.

\section{Metastasis to Pituitary Adenoma - A Case Report.}

C. RAO, F. SCHNEIDER, J. ASTARLOA, B. SHTEYNGART and P. CHEN (Kings County, State University Health Science Center and Brooklyn Hospital, Brooklyn, NY)

Tumor to tumor metastasis is an uncommon occurrence. The most common intracranial neoplasm that receives metastasis is meningioma. Metastasis to a pituitary adenoma is a rare event, only thirteen cases reported thus far. The commonest primary sites of origin for these metastasis to pituitary adenomas were breast (3), lung (3) and stomach (2). Only one case of small cell carcinoma of lung metastatic to pituitary adenoma has been reported previously. We report a second case of small cell carcinoma of lung metastatic to pituitary adenoma. This was a 61-year-old black male with a one year history of small cell carcinoma of lung who presented with loss of vision. A CT scan revealed a sellar-suprasellar mass. A presumptive diagnosis of metastatic carcinoma was made and radiotherapy was given. Two weeks later the patient expired with unabated carcinoma and pneumonia. At autopsy, the sellar-suprasellar tumor was shown to be a pituitary adenoma with sizable areas of metastatic carcinoma. The immunoperoxidase stains for a battery of pituitary hormones revealed scattered ACTH positive cells in the adenoma, the metastatic carcinoma cells being negative. Cytokeratin positivity was seen in both tumors.

6.

Universal Absence of Merlin, but not ERM Family Members, in Schwannomas.

A.O. STEMMER-RACHAMIMOV, L.B. JACOBY, J.F GUSELLA, V. RAMESH and D.N. LOUIS (Molecular NeuroOncology Laboratory and Molecular Neurogenetics Unit, Massachusetts General Hospital and Harvard Medical School, Boston, MA)

Neurofibromatosis 2 (NF2) gene mutations and chromosome $22 q$ loss have been demonstrated in the majority of sporadic and NF2-associated schwannomas, but many schwannomas fail to demonstrate genetic evidence of biallelic NF2 gene inactivation. In addition, the role of the related ERM family members (ezrin, radixin and moesin) remains unclear in these tumors. We therefore studied expression of NF2-encoded merlin as well as ezrin, radixin and moesin in 22 vestibular and peripheral schwannomas which had been evaluated for NF2 mutations and chromosome $22 q$ loss. Western blotting and immunohistochemistry with antibodies directed against the amino and carboxy termini of merlin demonstrated loss of merlin expression in all studied schwannomas, including twelve tumors lacking genetic evidence of biallelic NF2 gene inactivation. Western blotting with antibodies directed against ezrin, radixin and moesin, however, showed expression of these proteins in all schwannomas. In addition, immunohistochemistry with antibodies to moesin revealed widespread expression in tumor and endothelial cells. These data indicate that the specific loss of merlin is universal to schwannomas and is not linked to loss of ezrin, radixin or moesin expression. (Supported by NIH Grants NS24279 and CA51410, and by a grant from the U.S. Army.)

7.

\section{Image Analysis of Proliferation in Meningiomas.}

D.H. GEORGE, and J. INVIK (Royal University Hospital, Saskatoon)

Meningiomas have a tendency to recur that may be difficult to predict by histology. An increased recurrence risk is associated with atypical meningiomas, defined by numerous mitoses, necrosis, cellularity, and cytological atypia. Malignancy is defined by marked atypia and cerebral invasion. Although useful, the definition of atypia and malignancy are partially subjective. In an attempt to develop more objective criteria for prognostication in meningiomas, we have begun measurements of the proliferation index (PI) of these tumors on MIB-1 stained sections using a semiautomated image analysis system (Optimas). We have begun by considering the risk of early ( 5 year) recurrence. We retrospectively studied 24 meningiomas initially treated by surgery only, and with at least 5 years follow-up. Histologically, one was malignant, 4 were atypical, and 19 benign. Three recurred within the first 5 years; there were no deaths. Of these, one was malignant, one atypical, and one benign. The mean non-recurrent PI was 0.58 (range 0.05-2.00). The mean recurrent PI was 2.51 (0.9-5.02). PI of recurrent tumors correlated with rapidity of recurrence. Three of 9 with $\mathrm{PI} \geq 0.9$ recurred. One of 5 benign tumors with $\mathrm{PI} \geq 0.9$ recurred, at 58 months. One of 4 atypical meningiomas recurred, with PI of 5.02. We conclude that PI of meningiomas can be a sensitive but non-specific predictor of early recurrence. Specificity is improved by the combination of atypia and very high PI.

8.

\section{Schistosomiasis Presenting as a Brain Tumour.}

I.R. MACKENZIE, Z.H. KISS and A. GUHA (The Toronto Hospital, Toronto)

Schistosomiasis is a metazoal infection caused by trematodes of the genus Schistosoma. Man is the definitive host while fresh water snails are the intermediate host. Schistosomiasis is endemic in tropical countries and human disease is usually 
caused by $S$. mansoni (South America and Africa), S. haematobium (Africa) or S. japonicum (Asia). Neurological involvement is uncommon but may occur when parasite eggs reach the CNS and elicit an inflammatory response. S. mansoni and $S$. haematobium usually involve the spinal cord producing either an acute transverse myelitis or chronic myelopathy. Cerebral involvement is more common with $S$. japonicum and may manifest as acute meningoencephalitis or as a focal tumour-like mass.

We describe an unusual case of $S$. mansoni presenting as an intracerebral mass lesion. A 26-year-old man complained of episodic, right-sided, fronto-temporal headaches for three months. The headaches were often accompanied by visual abnormalities consisting of blurring of vision and "seeing flashing lights". Past medical history was unremarkable. Social history revealed that he had previously lived on a farm in central Brazil and had immigrated to Canada four years prior to presentation. General physical and neurological exam were unremarkable. Routine laboratory investigations and chest X-ray were normal. Head CT and MRI identified an enhancing lesion with surrounding edema and mild mass effect in the right occipital lobe. A stereotactic biopsy showed intraparenchymal granulomatata surrounding Schistosoma eggs.

9.

\section{Spinal Angiolipoma: Clinicopathological and} Ultrastructural Study of Two Cases.

S. ALOWAMI, L.C. ANG, J. CHAN, and D.W. ROWED (Sunnybrook Health Science Centre and University of Toronto, Toronto)

Angiolipomas are benign tumours composed of mature adipocytes admixed with vascular channels. These tumours are found in the subcutaneous tissue, muscle, bone and kidney. In the spinal canal, these tumours are predominantly seen in females and are located in the epidural space especially at the mid-thoracic level. We report two cases of thoracic extradural spinal angiolipomas in two elderly females ( 73 years and 78 years). Both patients presented with features of spinal cord compression which was relieved by surgical resection. In both cases, the diagnosis of angiolipoma was only apparent on histological examination. Ultrastructurally, both tumours appeared to have a decrease in Weibel Palade bodies in the endothelial cells as well as long-spacing collagen in the extracellular matrix. The decreased in Weibel Palade bodies has been previously described in angiolipomas elsewhere. Long-spacing collagen is classically seen in Schwannoma and structures such as the Descemet's membrane but its presence in angiolipomas has not been previously documented.

10.

Leigh's Disease: Presentation Mimicking Motor Neuropathy and Confined to Spinal Cord.

E.S. JOHNSON, F. BAMFORTH and S. BAMFORTH (University of Alberta, Edmonton)
As protean as the manifestations of Leigh's disease are, this report documents a unique presentation mimicking a motor neuropathy in an 18-month girl. At 14 months the patient lost strength in the legs after MMR immunization; Guillain-Barre syndrome was suspected and a course of immune globulin therapy given, begetting a dramatic improvement. However, at 17 months she became irritable, lethargic, and developed progressive weakness in all limbs terminating in quadriplegia over three weeks. Nerve conduction studies, electromyography, and MRI scan of the brain and cervical cord were normal; muscle biopsies showed type II atrophy. Further deterioration was heralded by onset of renal tubular acidosis, hyperammonemia, elevated CSF lactate $(5.3 \mathrm{mmol} / \mathrm{L})$, and later lactic acidosis $(7.1 \mathrm{mmol} / \mathrm{L}, \mathrm{N}=0.5-2.2)$. At autopsy the characteristic spongiotic degeneration of Leigh's disease was confined to the spinal cord with bilateral involvement of the corticospinal tracts, anterior columns and contiguous gray horns, and focally the posterior columns. Minor involvement of the pyramids and dorsal nuclei of the vagi was noted in the caudal medulla. In the peripheral nerves examined a few fibres undergoing Wallerian degeneration were observed in the left femoral nerve. Normal activity of pyruvate dehydrogenase in fibroblast cultures and of complexes I to IV in cytochrome oxidase in muscle from the biopsy specimen were assayed. However, mitochondrial DNA mutations could not be excluded because of insufficient tissue for further analysis.

11.

\section{Early and Sustained Increase of Gelatinase B (MMP-9) Activity after Stroke.}

A.W. CLARK, S.S. BOU, K.R. CHAPMAN, C.A. KREKOSKI and D.R. EDWARDS. (Departments of Pathology and Clinical Neuroscience and the Neuroscience Research Group; and the Department of Pharmacology and Therapeutics, University of Calgary, Calgary)

Cerebral edema typically peaks two days after stroke and is a potentially lethal complication. Gelatinases are a subgroup of the matrix metalloproteinases (MMPs), enzymes involved in remodelling extracellular matrix. Gelatinases A (72 kDa, MMP-2) and B (92 kDa, MMP-9) degrade type IV collagen and fibronectin of vascular basement membrane, and therefore might exacerbate post-stroke cerebral edema. In the present study, we used gel zymography to measure activities of gelatinases $\mathrm{A}$ and $\mathrm{B}$, comparing infarcted and non-infarcted tissue from eight postmortem human brains at post-stroke intervals of less than 2 hours to more than 6 years. Gelatinase $B$ activity showed no change in the shortest post-stroke interval but was markedly elevated in subjects dying at 2-5 days; and remained elevated for many months after the event. Gelatinase A activity showed subtle changes in the 2-5 day interval, which became marked and significant in the cases dying at 4 months and later. The findings indicate that gelatinase $A$ and $B$ activities increase in human brain following stroke, gelatinase B in the interval in which edema is evolving, gelatinase A somewhat later, with both activity increases persisting for months. Early inhibition of the activity of gelatinase $\mathrm{B}$ may be useful in controlling the edema associated with cerebral ischemia. 
12.

\section{Promoting the Survival of Human Fetal Dopamine Neurons in vitro: Implications for the Treatment of Parkinson's Disease.}

J. WOULFE, M. LEBLANC and R. HAMMOND (London Health Sciences Centre, University of Western Ontario, London)

Parkinson's disease is a neurodegenerative condition, the pathological hallmark of which is the progressive loss of mesencephalic dopaminergic neurons. There has been considerable interest recently in the role of certain neuronal trophic factors in the pathogenesis as well as the potential therapy of Parkinson's disease. Brain derived neurotrophic factor (BDNF) and glial cell line derived neurotrophic factor (GDNF) are especially promising in this regard. These factors undergo selective retrograde transport in rat nigrostriatal dopamine neurons and enhance the survival of rat midbrain dopamine neurons under a variety of conditions. Consequently, they have been regarded as selective, targetderived neurotrophic factors for this neurochemically-defined cell population. The neuropeptide neurotensin (NT) also shows promise as a potential trophic factor for midbrain dopamine neurons. We examined the effects of BDNF, GDNF, and NT on the survival and differentiation of human fetal catecholaminergic neurons. Primary cultures of human fetal CNS derived from 16-20 week gestation fetuses were grown in unsupplemented serum free medium or in medium supplemented with $10 \mathrm{ng} / \mathrm{ml}$ BDNF, 10 $\mathrm{ng} / \mathrm{ml}$ GDNF or $10 \mathrm{nM}$ NT alone or in various combinations for durations of 1, 2, or 4 weeks. Immunohistochemistry for tyrosine hydroxylase was employed to monitor the survival of dopamine neurons. Supplementation of the medium with BDNF, alone or in combination with the other factors, substantially enhanced the number of tyrosine-hydroxylase immunoreactive neurons at all time points examined. These results support a role for BDNF in the maintenance and survival of human dopaminergic neurons. They will be discussed in the context of in vitro and animal model strategies designed to further elucidate factors involved in the development and survival of dopamine neurons.

13.

\section{Can Tumour Characteristics of Glioblastoma Multiforme Predict Survival?}

J.N. SCOTT, N.B. REWCASTLE, P. BRASHER and P. FORSYTH (University of Calgary, The Foothills Medical Centre and Alberta Cancer Board, Tom Baker Centre, Calgary)

It is known that some patients with histologically confirmed glioblastoma multiforme (GBM) inexplicably survive longer than anticipated with current therapy. In an attempt to predict long term glioblastoma multiforme survivors (LTGBMS), this study addressed: 1) histological tumour characteristics, 2) treatment factors, and 3) a population based frequency. Long term was defined as surviving $\geq 3$ years. GBM was defined as a malignant glial neoplasm with increased vascularity, endothelial proliferation and necrosis with or without palisading. Excluded were any GBMs with features of oligodendroglial or ependymal origin. Over a 16 year period $689 \mathrm{GBMs}$ were identified through the Alberta Cancer Registry of which 17 (2.5\%) qualified as LTGBMs. Each index case was compared with 3 matched GBMs for age, sex and age of disease onset. LTGBMs tended to have less mitoses per 5 high power fields (8.9 compared to controls 14.7) and a lower Ki67 proliferation index (10.6\% compared to controls $21.5 \%)$. Of the Southern Alberta cohort $(\mathrm{N}=5)$, LTGBMs were significantly younger than controls $(\mathrm{p}=0.0001)$ and had a higher KPS at diagnosis than controls $(p=0.001)$. Other predictive factors were not identified.

14.

\section{The Immunopathology of Gangliogliomas.}

S. SMITH, S. LOWNIE and R. HAMMOND (London Health Sciences Centre and University of Western Ontario, London)

Gangliogliomas are lesions of low malignant potential whose presentation, morphology and natural history are compatible with a developmentally acquired "tumour". Lymphoid infiltrates are a routine finding on which little significance has been placed in the past. A recently examined spinal cord ganglioglioma contained a prominent lymphoid infiltrate prompting a review of the nature of the inflammatory component in this entity.

A 30-year-old man experienced a 7 year history of a weak urinary stream. For the two years leading up to surgery, there was neurologic progression with increasing bowel and bladder dysfunction, sensory symptoms in the sacral dermatomes and leg weakness. MRI showed an intraaxial fusiform mass in the conus medullaris which was subsequently resected. What proved to be a ganglioglioma showed marked $\mathrm{T}$ and $\mathrm{B}$ cell infiltration and microglial activation. Ten recent cerebral examples showed more modest inflammation by comparison. The latter cases contained rare $B$ cells, variable mild to moderate $T$ cell infiltrates and moderate to marked microglial activation.

The present case stands out for the degree of inflammation as well as its composition. Furthermore, the crescendo of symptoms is best attributed to the inflammation whether by mass effect or by the chronic secretion of inflammatory mediators (cytokines, etc.) or both. This study confirms the commonality of inflammatory infiltrates in gangliogliomas, and characterizes the typical infiltrate as one of $\mathrm{T}$ cells and activated microglia. Finally, the present case suggests that inflammation may influence the tempo and severity of symptoms associated with gangliogliomas.

15.

\section{Prevention of HIV-1 gp120 Induced Neurotoxicity in Primary Serum-free Human CNS Cultures.}

R. HAMMOND and M. LEBLANC (London Health Sciences Centre and University of Western Ontario, London)

The mechanism of neurotoxicity associated with HIV-1 infection leading to AIDS dementia is unresolved. The HIV-1 coat protein gp120 has long been suspected as a key factor, a notion that has support from clinical and experimental data. It has been shown that monocytes (microglia/macrophages) mediate gp120-associated neurotoxicity and that its toxicity is linked to NMDA-receptor activation. Furthermore, there is evidence to suggest that the neuronal demise is calcium-dependent. 
We have developed and characterized a human CNS culture system to permit the long term maintenance of mature human neurons in a serum-free defined medium. The system provides a sensitive measure of sublethal neuronal injury to better approximate the subtle neuronal changes of AIDS dementia. Cultures exposed to gp120 constructs show neurotoxicity in the form of reduced microtubule associated protein (MAP) and class-III beta tubulin (C3T) expression as well as increased expression of glial fibrillary acidic protein (GFAP). Likewise, gp120 toxicity can be prevented with a variety of manipulations including calcium channel blockade. The system can act as a valuable tool for the screening of neuroprotective substances in experiments of HIV-I induced neuronal damage. (Supported by a grant to RH by the Bickell Foundation.)

16.

\section{The Distribution of Calretinin in the Human Thalamus at Autopsy.}

P.V. GOULD, M. FORTIN, M-C. ASSELIN and A. PARENT (Centre hospitalier Robert-Giffard et service d'anatomopathologie, CHA pavillon Enfant-Jésus, Québec).

The calcium-binding protein calretinin is abundant in the human brain, particularly in the GABAergic neurons of limbicassociated territories. We have previously documented the distribution of calretinin in the normal human thalamus on freezing microtome sections. In order to assess the preservation of thalamic calretinin-immunoreactivity after routine formalin-fixation we sampled twelve autopsy adult brains $(6 \mathrm{M}, 6 \mathrm{~F})$ at anterior and mid-thalamic levels. Patient ages ranged from 46 to 93 years, brain weights ranged from 1040 - $1552 \mathrm{gm}$, and postmortem intervals ranged from 3 to 18 hours.

All sections showed preservation of neuronal calretininimmunoreactivity in the medial limbic structures as well as in the intralaminar and reticular nuclei. Calretinin immunohistochemistry permitted positive identification of smaller nuclei which were inapparent on sections stained for cresyl violet/Luxol fast blue. These results suggest that calretinin immunostaining can assist in the neuropathologic assessment of the thalamus.

17.

Selective Atrophy of the Dentate Gyrus in a Familial Syndrome with Mental Impairment and Cataracts.

D.G. MUNOZ and A.J. HUDSON (London Health Sciences Centre and University of Western Ontario, London)

The mother and 6 of her 11 children in this French-Canadian family living in Northern Ontario were born with congenital cataracts. One of the children was mentally retarded; all the others demonstrated normal intellectual development, but developed during adult life a psychotic syndrome characterized by paranoid delusions, emotional volatility, and erratic behavior. Several patients had difficulty swallowing, and tended to choke and aspirate. Most deaths occurred during the 6th decade.

Autopsy was obtained in two cases. Both cases demonstrated selective, severe atrophy of the dentate fascia. Over $90 \%$ of the neurons in the granular cell layer were lost, and the remainder showed a swollen eosinophilic hyaline cytoplasm which did not take silver stains or expressed tau. Ultrastructurally these cells demonstrated deep invaginations of the nuclear envelope, and regularly arranged stacks of collapsed cisterns. Many oligodendrocytes in the white matter and the pencils of Wilson displayed intense cytoplasmic expression of B-crystallin. There was mild neuronal loss in the substantia nigra. The retina, cerebral cortex, and other parts of the brain were unremarkable. The B-crystallin gene was screened by single-strand conformation polymorphism and direct DNA sequencing. No mutations were found, indicating that the abnormality lies in another gene.

We propose that this is a new genetic syndrome, and the first human condition to demonstrate selective atrophy of the dentate gyrus.

18.

Wolfram Syndrome: Report of a Case with Neuropathologic Examination.

P. SHANNON ${ }^{1,2}$, V. JAY ${ }^{1}$, L. BECKER ${ }^{1}$ and J. DECK ${ }^{2}$ (University of Toronto Division of Neuropathology, 'Hospital for Sick Children and ${ }^{2}$ Office of the Provincial Coroner of Ontario, Toronto)

Wolfram syndrome is a familial disorder comprising diabetes insipidus, diabetes mellitus, optic atrophy and deafness (DIDMOAD). We report a patient who developed diabetes mellitus at age 6 years, central diabetes insipidus at age 7 years, and optic atrophy which progressed to blindness by age 12 years. She also had mild sensorineural hearing loss and childhood urinary retention and enuresis. Additional investigations showed normal thyroid, adrenal and gonadal function. CSF biochemistry and cytology were also normal. Her subsequent development and cognitive status were otherwise normal. Neurologic examination at age 30 years showed additional mild loss of vibration sensation and ankle jerk reflexes. She died suddenly at home at age 38 years; systemic autopsy revealed aspiration of gastric contents. The brain findings included neuronal loss in the supraoptic nucleus and in the lateral geniculate body, with atrophy of the optic radiations. The pons was atrophic with loss of neurons in the pontine nuclei and axonal loss in the cerebellopontine tracts. Atrophy and gliosis of the olfactory tract were also present. This is the fifth autopsied case reported in the world literature.

19.

\section{Nestin Expression in Developmental Brain Lesions.}

N. DUGGAL, M. LEBLANC and R. HAMMOND (London Health Sciences Centre and University of Western Ontario, London)

Nestin, a $200 \mathrm{kD}$ intermediate filament protein, is expressed by immature (stem) cells of neuroepithelial lineage that possess the ability to differentiate into both neurons or glia. It is strongly expressed in the human brain during early stages of development. As cellular maturation proceeds, nestin expression is progressively downregulated in the neuroepithelial cells where it is supplanted by more mature intermediate filaments (NF, GFAP). 
In human neoplasia, expression has been described in tumours of neuroepithelial origin including primitive neuroectodermal tumours and gliomas as well as meningiomas.

What is the significance of nestin in the regulation of deveopmental and neoplastic events in the human CNS? We looked for the expression of nestin in cortical dysplasias and dysembryoplastic neuroepithelial tumours (DNET). Nestin expression was found to be present in cells of radial glial morphology, in the oligodendroglial component of DNETs and in occasional ectopic neurons in cortical dysplasias. The continued presence of nestin expression in radial glia, oligodendroglia and neurons in these developmentally acquired lesions is noteworthy given their presumed origin and nature. As such, nestin may be a marker of neuroepithelial dysembryogenesis.

\section{Titles of Diagnostic Case Presentations}

1. Melanotic neuroectodermal tumor of the skull and meninges.

A. LAROSE and Y. ROBITAILLE (Montreal, PQ).

2. Brain stem encephalitis with demyelination due to Adenovirus infection in HIV+ AIDS.

D.F. UPHOFF and D. KNIBBS (Hartford, CT).

3. Glioblastoma multiforme: gliosarcoma versus epitheliod form?

R.J.B. MACAULAY and R. GRIEBEL (Saskatoon, SK).

4. Forme fruste of tuberous sclerosis. D.H. GEORGE (Royal University Hospital, SK).

5. "Onion bulb" formation in dorsal and ventral roots in association with multiple sclerosis.

W. HALLIDAY (Winnipeg, MB).

6. Microvasculitis associated with systemic lupus erythematosus.

J.M. BILBAO and S. COHEN (Toronto, ON).

7. Desmoplastic infantile ganglioglioma. D. ARMSTRONG (Houston, TX).
8. Ependymoma with cartilage formation on recurrence. A.G. LACSON, C.H. ROSSBACH, R. NUTALL and M. SUTCLIFFE (St. Petersburg, FL).

9. Intracranial plasma cell granuloma. D. KYDD and J. ROSSITER (Kingston, ON).

10. Varicella zoster vasculitis and meningoencephalitis. P. SHANNON, J.M. BILBAO and W. OZANNE (Toronto, ON).

11. (A) Pacinian corpuscles neuroma ("pacinioma"), (B) Neuromata of the adrenal gland. A. HAYNER-BUCHAN, A.D. DEL ROSARIO, J.D. DOLLAR and A.H. KOEPPEN (Albany, N.Y).

12. Late delayed radiation necrosis. C. ARMSTRONG and D. MUNOZ (London, ON).

13. Atypical limbic encephalitis. S. MURRAY and V. SANGALANG (Halifax, NS).

14. Moya-Moya syndrome. D.P. AGAMANOLIS (Akron, OH). 